27th EPS Conference on Contr. Fusion and Plasma Phys. Budapest, 12-16 June 2000 ECA Vol. 24B (2000) 1148-1151

\title{
Comparison of theory based transport models with ASDEX Upgrade data
}

\author{
G.Tardini, A. G. Peeters, G. V. Pereverzev, F. Ryter, J. Stober, \\ and the ASDEX Upgrade team
}

Max Planck Institut für Plasmaphysik, Boltzmannstrasse 2, D-85748 Garching, Germany

\section{Introduction}

Heat transport in steady state Asdex Upgrade discharges is compared with the predictions of theory based transport models. Three different models are applied in the simulations: two based on the combination of Ion Temperature Gradients (ITG) and Trapped Electron Mode (TEM) physics, the IFS/PPPL [1] and the Weiland model [2]; and one based on the Current Diffusive Ballooning Mode (CDBM) model [3]. The discharges for this study (parameters are given in Table 1) are selected to provide scans over heating power, density and plasma current, keeping two quantities fixed and varying the third. The ITG models in particular yield stiff temperature profiles, with the core temperature depending directly on the boundary condition. The scans are expected to be a good test of this stiffness because the edge temperature (pressure) is varied over a wide range through different physics effects. A low boundary pressure shot is included to complete the database.

Experimental data are obtained from the following diagnostics: electron temperature $\left(T_{e}\right)$ from the electron cyclotron emission; ion temperature $\left(T_{i}\right)$, plasma toroidal rotation $\left(v_{\text {tor }}\right)$ and effective charge $\left(Z_{\text {eff }}\right)$ from the charge exchange recombination spectroscopy; electron density $\left(n_{e}\right)$ from the combination of Thomson scattering and Lithium-beam. Auxiliary heating in the considered experiments is obtained through neutral beam injection (NBI).

\begin{tabular}{|l|c|c|cc|}
\hline \hline & $P_{N B I}(\mathrm{MW})$ & $I_{p l}(\mathrm{MA})$ & $n_{e}\left(10^{19} \mathrm{~m}^{-3}\right)$ & \\
\hline Standard & 5.0 & 1.0 & $6.0-8.0$ & \\
\hline Scans & 2.484 & 0.4 & 3.93 & 5.43 \\
& 4.952 & 0.6 & 3.97 & 5.86 \\
& 7.353 & 0.8 & 4.08 & 7.13 \\
& 9.965 & 1.0 & 4.16 & 8.07 \\
& 12.460 & 1.2 & 4.61 & 10.30 \\
& & & 5.24 & \\
\hline \hline
\end{tabular}

Tab. 1 Parameters of the selected Asdex Upgrade discharges

All simulations are performed with the ASTRA transport code (see [4]), keeping the densities equal to the measured profiles. The experimental temperatures at $\mathrm{r} / \mathrm{a}=0.8$ are taken as boundary conditions for the calculation, because the models are not expected to describe the pedestal region correctly. Current diffusion is calculated at every time step after neoclassical theory. Sawteeth are taken into account with a Kadomtsev full reconnection model, the period being experimentally determined. Heat diffusivities are the sum of the neoclassical and turbulent contributions. In all three models the stabilizing term arising from the $E \times B$ shear $\left(\omega_{E \times B}\right)$ is included. This shearing rate is obtained from the measured $v_{t o r}$, the neoclassical poloidal rotation $\left(v_{p o l}\right)$ and the pressure $\left(p_{i}\right)$ gradient 
27th EPS CCFPF 2000; G. Tardini et al.: Comparison of theory based transport models with ASDEX Upgrade ...

contribution from the modelled profiles.

\section{Results}

In Fig. 1 it is possible to recognize the proportionality between stored energy $\left(W^{\exp }\right)$ and total boundary pressure $\left(p^{e x p}(0.8)\right) . W^{\exp }$ is not measured directly but integrated from the experimental temperature and density profiles. In Fig. 1 (b) $W_{i}^{\text {exp }}$ is plotted against $p_{i}^{e x p}(0.8)$ showing the same linear behaviour. This simple proportionality is observed also
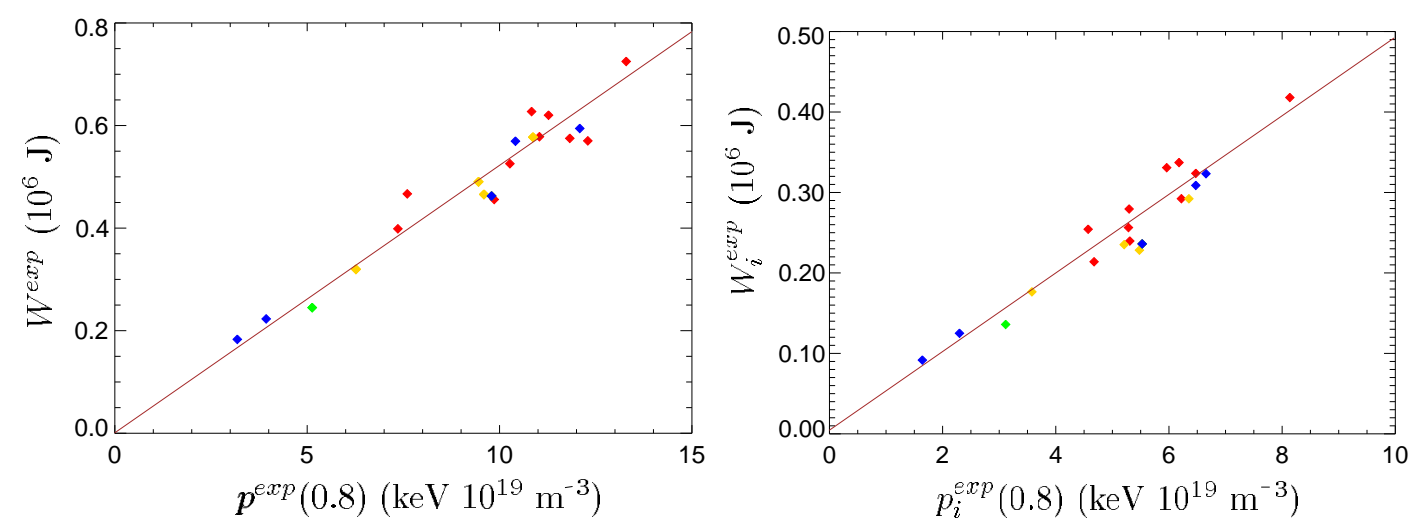

Fig. 1 Measured stored energy against $p^{\exp }(0.8)$; red points belong to the $n_{e}$, yellow to the $P_{N B I}$ and blue to the $I_{p l}$ scan; the green point is the low $p^{\text {exp }}(0.8)$ shot; (a) plasma energy, (b) ion energy

between core and boundary $T_{i}$ values, as shown in Fig. 2 (a). In all Figures 1 (a), (b) and 2 (a) the linear response is common for all scans. From Fig. 2 (b) follows that,
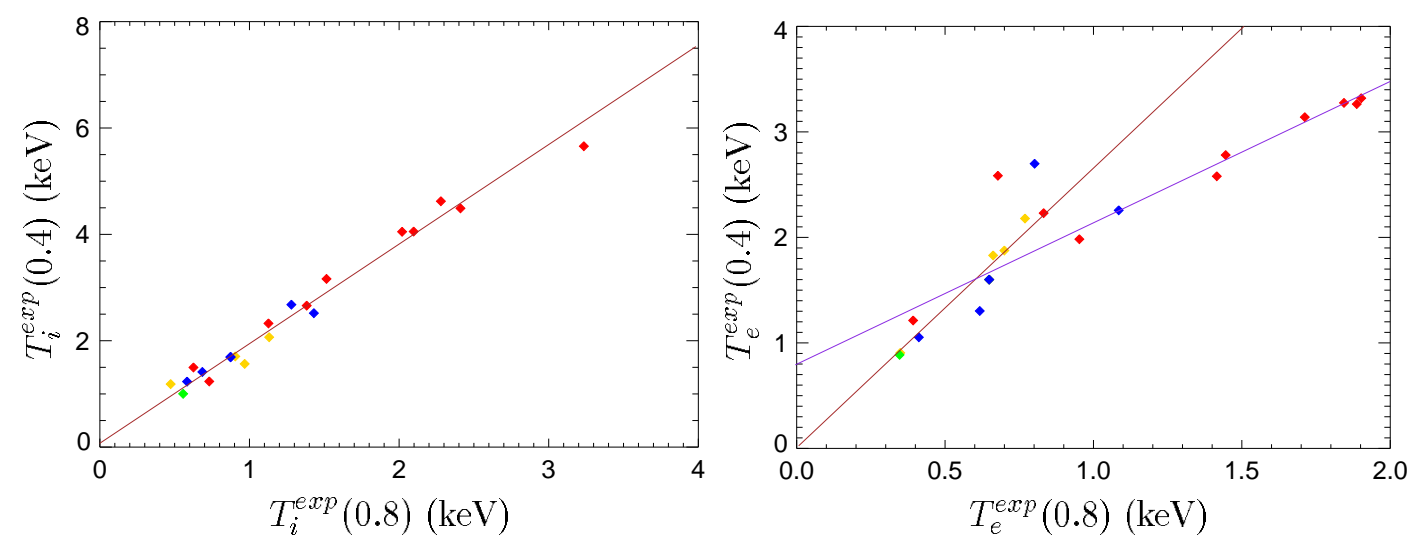

Fig. 2 Experimental core against boundary temperatures (colours like in Fig. 1); (a) $T_{i}^{\exp }(0.4)$ against $T_{i}^{\text {exp }}(0.8)$, (b) $T_{e}^{\text {exp }}(0.4)$ against $T_{e}^{\exp }(0.8)$

unlike $T_{i}^{e x p}$, for $T_{e}^{\exp }$ the ratio between core and boundary tends to decrease slightly at high boundary $T_{e}^{e x p}$, and the data have more scattering than in the case of $T_{i}^{e x p}$, but more experimental points would be required.

The corresponding plots for simulated temperatures are shown in Fig. 3. Both the ITG based models show a linear relation between $T_{i}(0.4)$ and $T_{i}(0.8)$, and the IFS/PPPL shows a slightly lower ratio $T_{i}(0.4) / T_{i}(0.8)$ than the Weiland model, that has a better quantitative agreement with the experimental data. CDBM seems to have two different linear trends, one at low and one at high boundary temperatures. For high values the interpolating straight line has a lower derivative and does not match the origin. 
27th EPS CCFPF 2000; G. Tardini et al.: Comparison of theory based transport models with ASDEX Upgrade ...
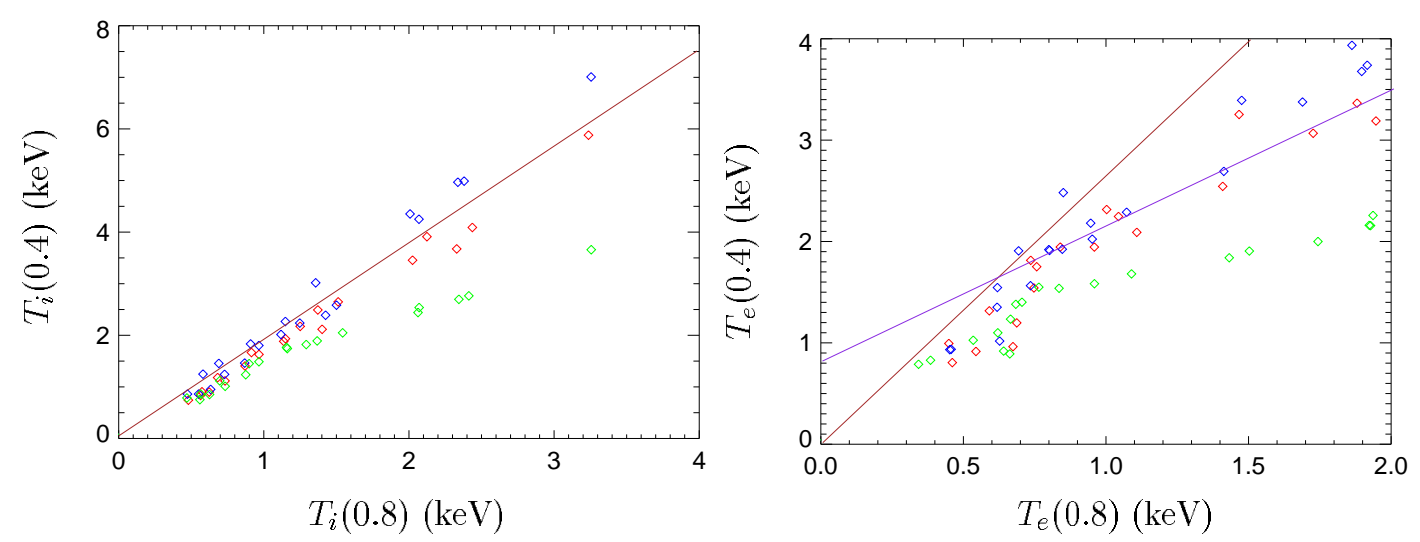

Fig. 3 Simulated core against boundary temperatures. Red points are related to the IFS/PPPL, blue to the Weiland and green to the CDBM models; the lines are the same as in Fig. 2; (a) $T_{i}(0.4)$ against $T_{i}(0.8)$, (b) $T_{e}(0.4)$ against $T_{e}(0.8)$

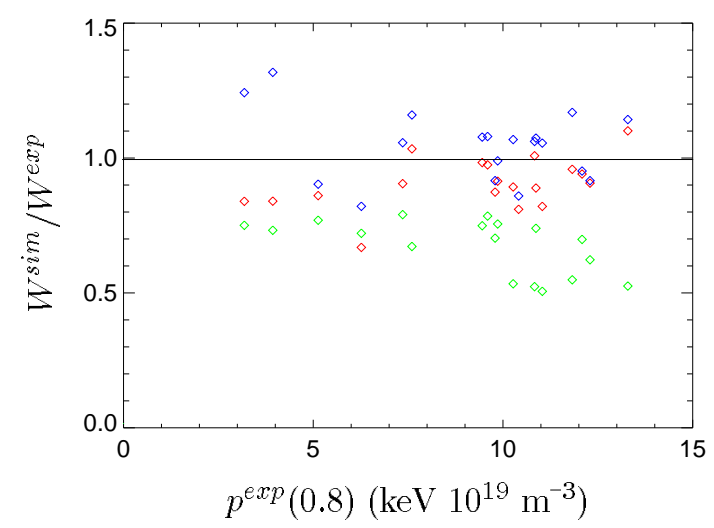

Fig. 4 Ratio of calculated to measured stored energy against $p^{\exp }(0.8)$ for the three models; colours like in Fig. 3

Figures of merit are introduced as defined in [5], to evaluate the reproduction of the experimental data by the models. In particular, the ratio of the calculated to the measured plasma energy is plotted in Fig. 4. Both experimental and modelled energies are taken for $0.2 \leq \mathrm{r} / \mathrm{a} \leq 0.8$. The boundary pressure extended to the whole volume represents about one half of the plasma energy. Therefore the product of the volume between 0.2 $\leq \mathrm{r} / \mathrm{a} \leq 0.8$ times $p(0.8)$ was subtracted, to consider only the part of energy due to the model.

The IFS/PPPL and CDBM models tend to underestimate plasma energy, the Weiland model yields in average the best reproduction of the data; however it has a larger scattering than the IFS/PPPL model. Both ITG models predict the experimental energies within $20 \%$. The deviations of the CDBM model exceed almost always $20 \%$ and become larger at high boundary pressure: the average predicted energy gets lower and the scattering gets larger.

\section{Discussion}

Profile stiffness means that heating power does not influence the $T_{i}$ profile, which tends to keep its shape. Heating power as well as $n_{e}$ and $I_{p l}$ variations can only change the profiles indirectly, by changing them in the region where the profiles are not stiff. Since the selected discharges have almost flat $n_{e}$ profiles, stiff temperature or pressure 
27th EPS CCFPF 2000; G. Tardini et al.: Comparison of theory based transport models with ASDEX Upgrade ...

profiles are almost equivalent. ITG models predict stiff $T_{i}$ profiles because $\chi_{i}$ has a steep increase if $R / L_{T i}$ gets larger than $R / L_{T c r}$, and drops to the neoclassical value below $R / L_{T_{c r}}$. Therefore the ratio $\nabla T_{i} / T_{i}$ tends to remain close to its critical value, which does not vary much from shot to shot within our database. If the boundary $T_{i}$ increases, $\nabla T_{i}$ increases proportionally and the whole profile is enhanced by the same factor. Including the stabilizing term $\omega_{E \times B}$ in ITG based models enhances the treshold of turbulent transport, but does not change the predicted stiffness. The IFS/PPPL model is particularly stiff: for typical Asdex Upgrade parameters it gives $\frac{\partial \chi_{i}}{\partial R / L_{T c r}} \approx 20 \mathrm{~m}^{2} / \mathrm{s}$. Therefore, the small deviations from a linear relation in different experimental conditions (see Fig. 3) arise from differences in $R / L_{T c r}$ or $\omega_{E \times B}$ profiles. The Weiland model is less stiff by about a factor 10 , but the ratio of core to boundary $T_{i}$ is still almost independent on the plasma parameters in the considered ranges (see Fig. 3).

Profile stiffness in Asdex Upgrade discharges is observed, as follows from Fig. 1. $W^{\exp }$ is an integrated quantity and may yield in principle a linear response to changes in $p^{e x p}(0.8)$ even if the profile is not uniformly enhanced by the same factor. But it is significant that all scans show the same linear trend, in spite of the different underlying physics. Furthermore, $T_{i}^{e x p}$ profile show the same features of stiffness (Fig. 2) and again for each scan. None of the three models predicts particularly stiff $T_{e}$ profiles, as it can be seen from Fig. $3(b)$; this is in qualitative agreement with the measured values in Fig. 2 (b), but the quantitative agreement is better for the ITG based models.

Fig. 3 shows that for $T_{i}(0.8)$ below $1.2 \mathrm{keV}$ the models can not be distinguished. Indeed, under certain experimental conditions a non-stiff model can reproduce a linear response of the core to boundary temperature enhancement: in the ITG picture heating power leads to higher $p_{i}(0.8)$, and thus indirectly to higher core $p_{i}$, but in the experiments both edge and core are heated, which makes difficult to distinguish between the different physics. Also the boundary density, the density value which influences the temperaure profiles in a stiff model, in the experiments is usually coupled to the averaged density.

The linear relation between measured core and boundary temperatures is valid over the whole considered range. The ITG models predict this behaviour, the CDBM model does not for $T_{i}(0.8)$ larger than $1.2 \mathrm{keV}$. Also the plasma energy is underestimated by the CDBM model, and drops to even lower predictions in the high pressure range. To state these trends it is necessary to extend the database, in particular in the low density range.

\section{ACKNOWLEDGEMENTS}

Special thanks to Drs. Weiland, Dorland and Mikkelsen for making available the subroutines containing the models employed in this study.

\section{References}

[1] M. Kotschenreuther, W. Dorland et al, Phys. Plasmas 2, 2381 (1995)

[2] H. Nordman, J. Weiland, A. Jarmen, Nuclear Fusion 30, 983 (1990)

[3] S.-I. Itoh, K. Itoh et al.,Plasma Phys. Control. Fusion 38, 1743 (1996)

[4] G. Pereverzev et al., IPP 5/42 (1991)

[5] J. W. Connor et al., IAEA conference Montreal, 935 (1996) 